\title{
Turismo en las ciudades históricas: la ciudad sostenible
}

\author{
$M^{\mathrm{a}}$ Teresa Pérez Cano \\ Departamento de Urbanistica y \\ Ordenación del Territorio \\ Universidad de Sevilla
}

\begin{abstract}
Resumen
El artículo aborda desde una perspectiva urbanística, la falta de relación entre los procesos urbanos contemporáneos y la conservación del patrimonio urbano y territorial. Se pone de manifiesto el derroche de recursos que consume la sociedad más desarrollada, y la escasez de instrumentos teóricos, técnicos y legislativos de aplicación real para evitarlos.
\end{abstract}

\section{Palabras clave}

Urbanismo / Sostenibilidad / Turismo / Patrimonio / Ciudad Histórica / Territorio

Todo el mundo entiende como obvio, que el equilibrio económico consiste en gastar como máximo lo que se ingresa, o que sobre la base de determinados ingresos se establece un nivel de vida. Sin embargo este razonamiento que funciona bien al nivel de economía familiar, ni siquiera se plantea hasta ahora a escala urbana. Como ciudadanos, amparados en el anonimato de la masa, consumimos, gastamos, derrochamos, sin reponer nunca la despensa, como si los recursos fueran inagotables.

\section{El concepto de sostenibilidad}

La Sostenibilidad se acuña en la Cumbre Mundial del Medio Ambiente de Río de Janeiro en 1992. Allí líderes de 172 países se pusieron de acuerdo en pen- sar que "la salud de la naturaleza era esencial para el bienestar y la supervivencia de la humanidad". En el campo de lo urbano esto se traduce en el diseño, desarrollo y gestión de comunidades urbanas sostenibles o lo que algunos autores han dado en llamar Ecourbanismo

Sumidos en este lenguaje, las ciudades empiezan a considerarse como complejos ecosistemas artificiales, construidos en primera instancia para satisfacer las necesidades humanas, pero también -y no por ello es menos importante- con capacidad para proporcionar un biotopo a otras especies y cuyo impacto sobre el entorno natural debe ser cuidadosamente gestionado.

Por fin ecología y tecnología tendrán que andar de la mano. Se nos abre un nuevo horizonte: La ecotecnología permitiría hacer un uso más racional de las fuentes de energía renovables y no renovables. El reciclaje de los residuos sólidos y líquidos, la utilización de fuentes alternativas de energía, creación de microclimas... o la convergencia de tecnologías en el campo de la informática que permitan reducir el número de viajes... con el consiguiente ahorro de tiempo y combustible, disminución de la contaminación del tráfico y del estrés. La técnica puesta al servicio de una sociedad equilibrada, y no al contrario, como propiciatoria de desequilibrios.

El desarrollo urbano sostenible mantiene la calidad general de vida, asegura un acceso continuado a los recursos naturales y evita la persistencia de daños ambientales.

De todos es conocido el proverbio indio que nos señala que la Tierra no es una herencia a nosotros de nuestros padres, sino un préstamo temporal que nos han hecho nuestros hijos. Nosotros somos simplemente los que la tutelamos hasta que ellos se hagan adultos. En ese mismo sentido, el desarrollo sostenible es aquél que satisface las necesidades de la generación actual, sin comprometer la capacidad de las generaciones futuras para satisfacer las suyas propias. Es una cuestión de solidaridad intergeneracional. 


\section{El turismo sostenible será el que consiga el equilibrio entre "un medio turistico percibido por los usuarios en todo momento de alta calidad (lo que incluye la protección del medio natural y del patrimonio histórico) y una demanda constante". El problema es que se subordina la sostenibilidad al mercado}

Pero cómo se mide esta necesidad que tiene cada generación. Porque, es evidente, que el gasto en naturaleza no es igual en todo el planeta. Éste varía en el tiempo y en el espacio. La capacidad que tenemos hoy en día de esquilmar un territorio con urbanizaciones residenciales -incluso de segunda residencia-, de destruir un paisaje por la inadecuada colocación de un edificio, o el derroche en el consumo de agua que se hace necesario para mantener en nuestro clima un campo de golf, son ejemplos que nos ponen en evidencia que las necesidades no son para todos las mismas. Se hace preciso un marco de entendimiento, en el que podamos comenzar a trabajar.

Para ello se introdujo el concepto de capital a transferir de generación en generación. Este capital no es homogéneo, tiene al menos tres componentes: el capital artificial -físico- elaborado por el hombre (edificios, infraestructuras...), el capital humano de carácter muchas veces inmaterial (ciencia, conocimiento, técnicas, tradiciones...) y el capital natural (diversidad biológica, aire puro, agua...). Siguiendo este símil económico, cada generación debiera vivir de los intereses generados de la herencia recibida y nunca tocar el propio capital principal. Aún estando de acuerdo con esta filosofía, se plantean cuestiones tales como si son y por tanto se deben de mantener como capitales independientes entre sí: sostenibilidad fuerte. $\bigcirc$ dependientes, o sea, se puede consumir más de alguno de los tres capitales, siempre que el cómputo total entre todos sea siempre el mismo: sostenibilidad débil.

Otra forma de medir la sostenibilidad o el gasto que hacemos de nuestro capital es lo que se ha dado en llamar Huella Ecológica. Este indicador - de gran utilidad para el Urbanismo- mide la superficie de suelo productivo que necesitamos para satisfacer nuestras demandas materiales y de energía. La sostenibilidad se alcanzará cuando la superficie por persona de lo que gastamos (que no tiene porque ser lo mismo de lo que necesitamos) sea igual o menor que la superficie que nos corresponda como ser humano (resultado de dividir la superficie útil del planeta entre todos los habitantes del mismo).
Este cálculo se ha realizado para Andalucía y no deja de ser descorazonador. La huella ecológica del andaluz medio es de 5,53 hectáreas, lo que duplica con creces el valor del territorio andaluz. Dicho de otra forma, Andalucía necesitaría otro territorio igual al suyo vacío para satisfacer sus necesidades materiales y de energía. Es hora pues de pensar qué estamos haciendo.

\section{Ciudad histórica y sostenibilidad}

En el terreno del urbanismo, la sostenibilidad se ha estudiado muy poco, aunque son muchos los planes que se consideran a sí mismos sostenibles, como si ello fuese simplemente un adjetivo, sin más compromisos. Cuando se ha abordado realmente, se ha hecho desde la corriente del ecourbanismo. Son proyectos o planes donde se intenta controlar los aspectos que harían posible que nuestras ciudades fuesen más sostenibles, que hiciesen posible que ese consumo de capitales fuera menor, para poder así reducir nuestra huella ecológica. Están por tanto relacionados con el control sobre la movilidad, la convivencia de usos, el consumo de los recursos, la revitalización de tejidos históricos, la mejora de los canales de participación, de la calidad urbana, alternativas al espacio turístico como las llamadas ecovacaciones, telepueblos, etc. En España contamos con muy pocos ejemplos completos que aborden y pongan en prácticas estas cuestiones. Veremos que hay mucho por hacer.

\section{La movilidad}

El transporte emplea, hoy día, la cuarta parte de la energía total consumida por los seres humanos. Es por tanto una cuestión clave para el desarrollo sostenible de las urbes, la generación de modelos urbanos que reduzcan al máximo los desplazamientos.

La movilidad es una cuestión que afecta al concepto mismo de ciudad contemporánea. La ciudad históri- 


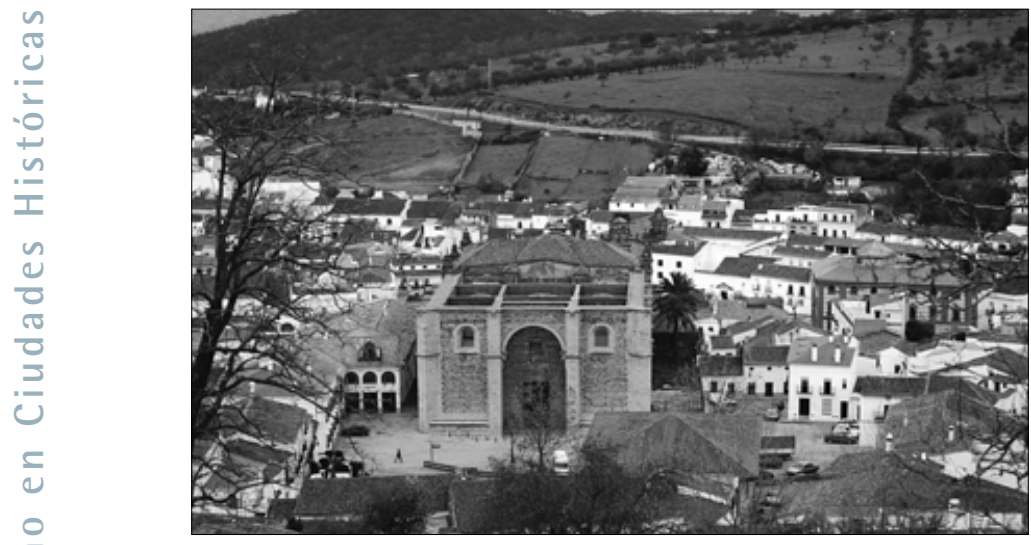

I. Los pequeños municipios mantienen una mejor relación entre patrimonio urbano y territorial que las grandes urbes. Vista de Aracena, (Huelva)

2. Interior de un corral en Granada.

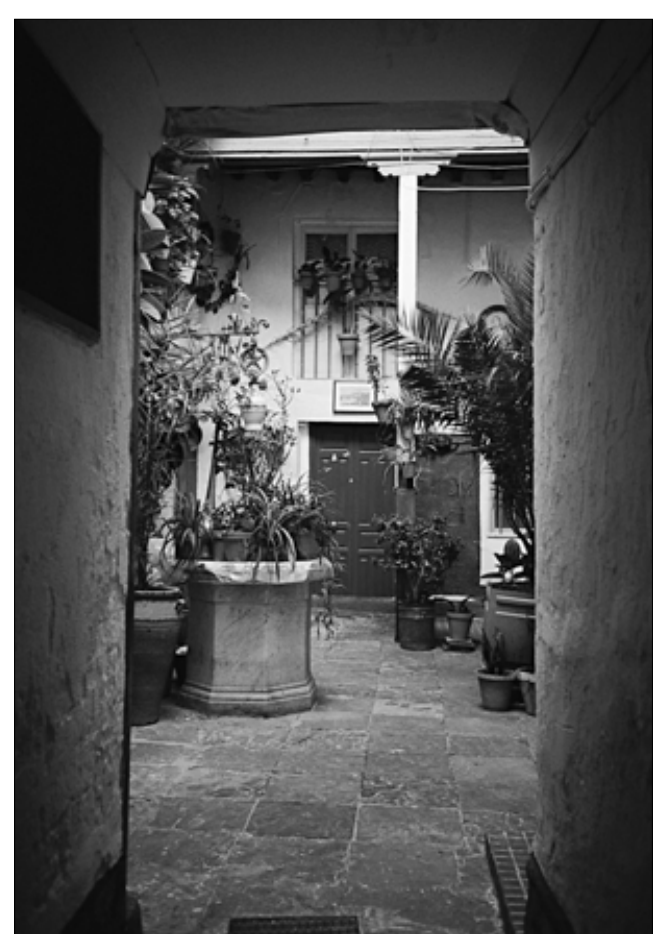

ca creció y se desarrolló hasta el siglo XIX sin la presencia de transporte motorizado. La mayoría de los desplazamientos en el interior de las poblaciones se efectuaban a pie o mediante la tracción animal. El ferrocarril como elemento para el transporte de personas y mercancías entre ciudades o su homólogo urbano -el tranvía -, generalizaron el acceso público al transporte. Pero la extensión de la ciudad moderna se basa en el "perfeccionamiento" de los medios de comunicación y sobre todo en la generalización del transporte privado. El movimiento funcionalista ampara su segregación de la ciudad en espacios para el trabajo, el ocio y la residencia, en el transporte privado. Las ciudades se piensan en cuanto a su trama, nudos de comunicación, ancho de las calzadas, etc., o sea tal y como la percibimos desde el espacio público, en función del número de vehículos que se prevé albergar. Romper esta inercia no es tarea fácil, sobre todo si hablamos al nivel de grandes ciudades y ciudades medias, y cuando no imposible en áreas metropolitanas o conurbaciones.

Todos sabemos que el vehículo privado produce contaminación y congestión -con las consiguientes pérdi- das económicas y humanas- y en su construcción se utilizan valiosos recursos naturales. En la ciudad histórica, ello se traduce en una clara pérdida de calidad urbana. Los efectos de la contaminación ambiental sobre el patrimonio edificado se pueden medir en muchos aspectos: deterioro material, físico, acústico.... A nivel del tejido histórico, rara vez éste permite el tránsito indiscriminado y el aparcamiento en la calzada de los vehículos. Las viviendas soportan con dificultad la adecuación de sus fachadas para la apertura de los huecos de garajes. Las aceras se ven reducidas o incluso suprimidas para ceder el máximo espacio al coche.

La planificación debe reconducir hacia las opciones más compatibles con la trama tradicional, para evitar la pérdida de sus valores. Debemos propiciar los transportes colectivos, invirtiendo la tendencia actual donde la mayoría de los desplazamientos se realizan en vehículos privados. En las grandes ciudades, opciones como el metro debería de ser casi incuestionables, sobre todo como alternativa pública para conectar el centro histórico con el resto de la ciudad. La ubicación de carriles para bicicletas, el diseño de un espacio urbano que favorezca el uso de la ciudad por los peatones... Pero también un nuevo urbanismo que planifique la ciudad interrelacionando los posibles usos urbanos, descentralizando funciones, favoreciendo la polinucleización, donde en definitiva se vean reducidas nuestras necesidades de utilizar cualquier transporte motorizado, y se recupere el espacio público como lugar de encuentro y convivencia.

\section{El capital artificial: los recursos}

Los edificios consumen el $60 \%$ de todos los recursos extraídos de la tierra. Nos podemos plantear la rehabilitación de edificios en la ciudad histórica, incluso como una cuestión de mero reciclaje, además de por sus valores artísticos, monumentales, tradicionales o domésticos. Materiales y sistemas constructivos ecológicamente entendidos nos deben servir para disminuir el consumo. Actuaciones apropiadas, como las planteadas por el Plan Especial del Conjunto Histórico de Córdoba inciden en esta línea. Es posible por tanto, desde un planeamiento y más concretamente desde unas ordenanzas, activar políticas de sostenibilidad.

Pero el consumo de recursos no hace más que empezar una vez que se ha construido el objeto arquitectónico. A partir de aquí, éste se convierte en un organismo vivo, que precisa de más energía para sernos útil. Los edificios consumen el $50 \%$ de la energía utilizada por el hombre. El resto que se gasta, se distribuye a partes iguales entre transporte e industria. Además, como efecto secundario, los edificios generan la mitad de las emisiones de $\mathrm{CO}^{2}$ a la atmósfera. Entraríamos - para ser sostenibles- en el campo de los diseños bioclimáticos, entendidos como aquellos que aportan soluciones arquitectónicas más compatibles con el medio ambiente. Por su propio diseño, orientación, etc. la arquitectura bioclimática derrocha menos energía. 
Los sistemas constructivos tradicionales de la ciudad histórica, estaban más adaptados - a falta de desarrollo tecnológico- para soportar por ejemplo los cambios de temperaturas estacionales. La anchura de los muros, el tamaño de los huecos de la carpintería, o la orientación de las estancias se racionalizaban. Hoy en día, la técnica lo hace todo posible, lo suple todo, aunque para ello necesiten cubrir déficits con apoyos extras en materiales y en consumo de energía: aislantes térmicos y acústicos, plásticos, asfaltos, y también ventilaciones forzadas, instalaciones de acondicionamiento, etc. por no hablar de otras adaptaciones tipológicas como la vivienda en altura (tras la aparición del ascensor) donde ya no hay marcha atrás.

Cobra ahora gran importancia la energía solar como complemento al consumo de energía eléctrica. Aunque en un futuro próximo debiera ser alternativa real, sobre todo en climas como el nuestro. Queda aún por solucionar el impacto visual y de deterioro del paisaje que producen los depósitos y los paneles solares.

Pero al hablar de recursos también nos referimos al suelo que consumimos, a los materiales, la energía, el agua o los residuos. Un sevillano medio, por ejemplo, consume 2,8 hectáreas de superficie productiva útil -tierra y mar- para satisfacer sus necesidades alimentarias. Gasta I, I hectáreas en energía, y otras 1,99 hectáreas las consume en superficie construida, embalses, escombros... El problema es que necesita 5,89 hectáreas y sólo dispone de 2,7 hectáreas de territorio productivo. Nuestra huella ecológica es negativa. ¿Qué significa ello?, que nuestro bienestar está sustentado en bienes ajenos, a los cuales accedemos por nuestra condición de preponderancia como primer mundo.

Si en los dos aspectos anteriormente tratados -movilidad y energía-, la ciudad histórica se sitúa con cierta ventaja respecto de la ciudad moderna, si tratamos el tema de un bien tan preciado como el agua, la cosa cambia. Tradicionalmente hemos construido ciudades que cuentan con sólo dos tipos de infraestructuras para el agua, la de la potable y la residual. Incluso, nos podemos encontrar hoy en día, sectores de ciudad histórica cuyo alcantarillado es defectuoso y coexisten un gran número de pozos negros. $\bigcirc$ una gran muestra de pueblos y ciudades medias que no depuran sus aguas residuales, siendo éstas vertidas al mar o a los ríos directamente.

Un desarrollo urbano sostenible se proyecta sobre la base de la optimización del ciclo del agua y cualquier tipo de agua -pluvial, potable o residual (grises y negras) - es utilizada como recurso. Desde la obtención de agua dulce por medios ecológicamente aceptables, pasando por la segregación de las aguas residuales, recogida y utilización de las aguas pluviales, retención de las aguas de escorrentías de tormenta o reposición del nivel freático.

Los residuos entendidos como recurso pueden generar energía. Podemos utilizarlos como materiales de construcción (los edificios en la antigüedad eran canteras de nuevas construcciones, por ejemplo), para la producción de abonos orgánicos, etc. No olvidemos que producimos una media de 1,6 Kg de residuos por persona y día, y debíamos hacer algo más que acumularlos y ocultarlos. La recogida selectiva como alternativa, es menos habitual en los sistemas más frágiles.

\section{La recualificación de la ciudad histórica}

Aunque en términos de sostenibilidad, en abstracto, se emplea más la idea de revitalización, para nosotros el término recualificación será sinónimo de recualificación urbana.

La ciudad se distingue principalmente de otros ecosistemas, en que su soporte físico -el biotopo-es en gran medida producto de la actividad humana. Artificial, consecuencia de cada cultura, que lo hace singular e irrepetible. En los proyectos urbanísticos de revitalización, se hace referencia a la vida, evocan la idea de insuflar nueva vida a un tejido urbano agotado. Se intenta combinar ciudad y ciudadanos, binomio que por definición debiera de ser inseparable, pero que paradójicamente, marchan con mucha frecuencia de forma independiente.

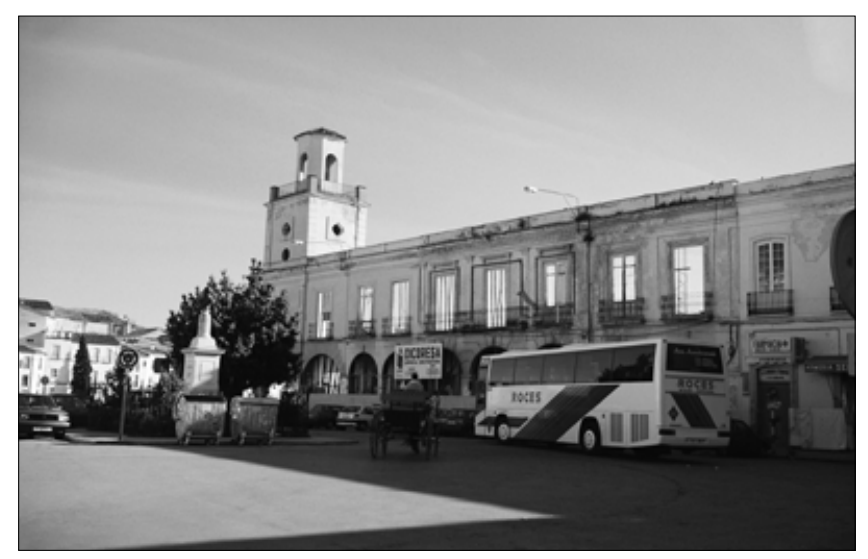

El tejido histórico tradicional no estaba mayoritariamente planificado. Es lo que podríamos llamar ciudad configurada orgánicamente por sus propios habitantes. Cada espacio tenía su razón de ser, era usado de forma real o simbólica de modo diferente por sus inquilinos, y ello lo identificaba y distinguía en primera instancia de otras partes de la ciudad, y a la postre de otras urbes.

Tendemos hoy en día, a convertir las ciudades históricas en biotopos vacíos, llenos de oficinas o de turistas, pero donde fracasa la relación habitante espacio construido. La imagen de progreso vinculada al urbanismo moderno, puede poner en peligro la rica cultura tradicional de vida en comunidad. Los centros históricos han sufrido en los últimos años procesos de expulsión de la población residente con bajo poder adquisitivo, donde tras un periodo de división parcelaria y aumento de la plusvalía, se incorporan nuevos agentes a la ciudad. Se altera la tipología y los modos de vida.
3. El espacio público de la ciudad al servicio de la movilidad del turista. Ronda, (Málaga). 

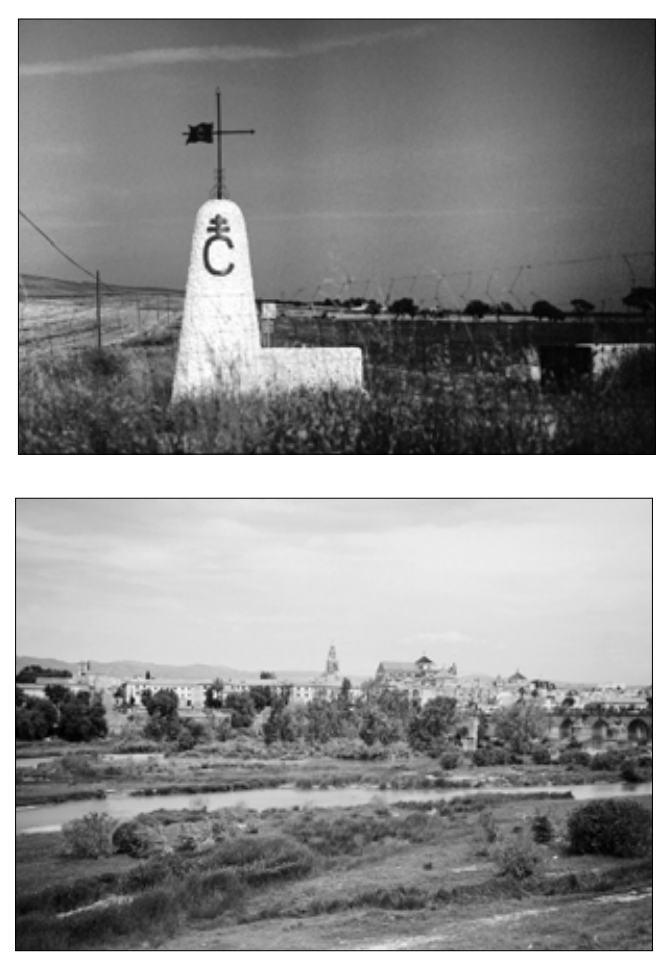

4. El mantenimiento actividades vinculadas a territorio, como la ganadera, propician su conservación. Jerez,

(Cádiz)

5. El Guadalquivir a su paso por la ciudad de Córdoba, guarda en algunos tramos el sabor de espacio no domesticado.
Uno de los objetivos que busca este urbanismo de nuevo cuño o ecourbanismo es el de proyectar -en un sentido amplio- comunidades humanas que proporcionen un entorno adecuado para las relaciones sociales y una vida comunitaria diversa y saludable.

Sin embargo, la ciudad histórica tradicional, los pueblos, el Sur, contenía tejidos que propiciaban las relaciones sociales, estimulaban la relación cara a cara entre las personas. El urbanismo sostenible apoya la rehabilitación de pueblos y centros urbanos, manteniendo la población residente, la defensa del patrimonio arquitectónico y la conservación de los entornos naturales, como única salida de futuro si queremos transmitir a nuestros hijos algo de nuestro presente.

En algunos casos, la combinación entre tradición y progreso puede conseguirse desde la mejora de las telecomunicaciones. Hay experiencias de este tipo, los Ilamados telepueblos más extendidos en Estados Unidos. Son empresas que se instalan junto con sus trabajadores en pueblos alejados en procesos de abandono, pero conectados con el exterior con las mejores telecomunicaciones. Se mejora así la calidad de vida de los trabajadores, a la empresa le resulta económico instalarse, pues es suelo barato, y se evita la desaparición del pueblo.

\section{La influencia fundamental del Turismo}

El turismo es la industria más importante del mundo. Genera, directa o indirectamente, el 10,7\% del producto interior bruto global y proporciona casi el I I\% de todos los empleos existentes en el mundo. En Andalucía, región turística por excelencia, estos datos son aún mayores proporcionalmente.
La actividad turística arroja uno de los índices de crecimiento más elevados y ha causado la destrucción de importantes ecosistemas en todo el mundo: playas, zonas costeras, montañas, humedales... Las previsiones indican que es un fenómeno en auge, que va a más. Aunque no todo es negativo, ya que el turismo también pueda servir para la integración social de áreas pocos desarrolladas.

Para su análisis, debemos tener en cuenta qué es el turismo. Se refiere siempre a una forma de residencia temporal, fruto de un viaje, un desplazamiento -esporádico o periódico -, por lo que la vinculación con el lugar del destinatario del producto es muy distinta de la que se desarrolla con el residente permanente. La ciudad funcional clásica, pensada para el trabajo, la residencia y el ocio, con las comunicaciones como telón de fondo que lo enlaza todo, pierde aquí uno de sus principales atributos. Podemos decir que son sectores de ciudad que se especializan en determinadas actividades, casi de forma monográfica, y abandonan otras.

Cabe siempre plantearse dos casos posibles, en función de la problemática tan distinta que se genera en el medio. Los primeros serían los asentamientos turísticos de nueva planta, sobre elementos naturales sin urbanización previa, ya sean como consecuencia de crecimientos de zonas urbanas existentes o de nueva planta. Los segundos, -y éste es el caso del turismo en la ciudad histórica- serían los asentamientos que se desarrollan sobre localizaciones preexistentes, o sea, los que afectan mayoritariamente a un tejido que se pensó para otro uso. Además, dentro de cada caso no es lo mismo el turismo que genera la segunda residencia, que la de los hoteles o las estructuras vacacionales.

El turismo aparece muchas veces como otra forma de explotar suelos, en principio con poca capacidad para el rendimiento productivo primario o secundario. Tiende a situarse en lugares de gran calidad paisajística y bonanza climática, de manera que se propicie el disfrute al aire libre, las prácticas deportivas, etc.

El modelo turístico es un proceso cuya evolución a través de distintas etapas es ya conocido. Comienza normalmente con el descubrimiento del sitio por parte de personajes o agentes exteriores. Continúa cuando se produce un apoyo del proceso, por parte de la iniciativa local, hasta su desarrollo y consolidación. A partir de aquí puede comenzar una etapa "crítica" de estancamiento, que puede conducir a la aparición de síntomas de saturación o degradación. Ésto puede determinar el declinar del fenómeno turístico y su traslado a nuevas zonas de interés, si no se toman antes medidas para su "rejuvenecimiento".

Cuando se habla de la necesaria planificación del turismo, para conseguir un turismo sostenible, se subrayan tres aspectos:

$1^{\circ}$. Ia utilización del medio natural de manera que los impactos negativos puedan ser corregidos sin dejar una huella permanente. 
$2^{\circ}$. el diseño y establecimiento de un producto turístico que tenga suficiente aceptación en el mercado para sostener los flujos turísticos a un mismo nivel a largo plazo.

$3^{\circ}$. el mantenimiento indefinido de la viabilidad económica del turismo, por medio de la adaptación constante al mercado.

La expansión turística ha conducido al progresivo deterioro del entorno turístico, tanto natural como construido. Como ya señaló Gerda K. Priestley "los destinos turísticos llevan consigo las semillas de su propia destrucción, a medida que se comercializan y pierden las características que originalmente resultaron atractivas para los turistas".

El turismo sostenible será el que consiga el equilibrio entre "un medio turístico percibido por los usuarios en todo momento de alta calidad (lo que incluye la protección del medio natural y del patrimonio histórico) y una demanda constante". El problema es que se subordina la sostenibilidad al mercado. Remediar las equivocaciones o corregir los excesos cometidos, empieza a ser fundamental para satisfacer las exigencias del turista a largo plazo.

Pero, es importante comprender que el turismo es una industria de servicios, y no simplemente construcción territorial de residencia. Por ello se debe ir más allá del estudio de las plusvalías añadidas al suelo, que la transformación comporta en la definición de costes de implantación y abordar los marcos para la implementación y el mantenimiento más amplio de la sostenibilidad global de los ámbitos territoriales.

Ello implica la comprensión global del proceso como recurso, el conocer los mecanismos de diagnóstico, el saber reconocer las oportunidades estratégicas, el saber instrumentar las soluciones.

La reciente Ley del Turismo de Andalucía, en su exposición de motivos, señala que su finalidad es la de "obtener el mayor provecho de los recursos turísticos, dentro del máximo respeto y cuidado de la cultura y tradiciones andaluzas y según las pautas del principio de sostenibilidad", ofreciéndose Andalucía como destino turístico integral. Propone la creación de una serie de instrumentos tales como Planes Generales del Turismo, Zonas de preferente actuación turística, Programas de recualificación de destinos, Municipio Turístico... que "pueda estimular un crecimiento ordenado y sostenible de nuestro sector turístico, salvaguardando el medio natural y nuestro patrimonio histórico y cultural".

La ordenación de la oferta turística pretende regular los servicios y establecimientos turísticos en general (actividades relacionadas con el ocio, alojamientos de todo tipo, restauración, intermediación, información...) en orden a establecer las "condiciones básicas que han de reunirse para respetary proteger el medio ambiente, el paisaje y la cultura andaluza".
Sin embargo, el incumplimiento de estas condiciones -infracciones que conlleven daños a los recursos turísticos, al medio ambiente o al patrimonio histórico- solo se considera una falta grave (intermedia entre las faltas leves o las muy graves) que caduca a los nueve meses de producirse y cuya multa no supera los dos millones de pesetas y la clausura del establecimiento por un periodo inferior a seis meses.

El urbanismo sostenible debería centrarse en restaurar zonas urbanizadas degradadas, antes que urbanizar las escasas y valiosas áreas naturales o agrícolas.

\section{Conclusión}

El urbanismo es una disciplina moderna, que como tal sólo cuenta con un siglo de existencia. Aunque llevemos produciendo ciudad más de veinte siglos, los últimos 60 años han sido los más destructivos desde el punto de vista de la sostenibilidad. Los planteamientos actuales del urbanismo frente al medio ambiente son comparables con los de la medicina en la Edad Media: miopes, parciales o llenos de miedo. Utilizamos instrumentos pensados para generar nuevas formas construidas -ciudad- desde la preponderancia del hombre sobre el medio que le rodea.

La reflexión del planificador ha pasado por distintas etapas. Hasta finales de los setenta, la principal preocupación del urbanista era crear ciudad. Los ochenta arrancan con una generación de planes que comienzan a interesarse por el Patrimonio Arquitectónico y en estos veinte años se ha instrumentalizado la intervención sobre la ciudad de manera cierta. Comenzamos ahora una nueva etapa.

El medio ambiente debe ser ahora el motor del planeamiento -sin olvidar lo aprendido en etapas anteriores. Los estudios de impacto ambiental, obligatorios para el planeamiento a gran escala, son sólo la punta del iceberg, pero fallan los mecanismos. Un ejemplo, aunque si bien es verdad que la mayoría de los Planes Generales que se redactan ahora son informados favorablemente por la Consejería de Cultura, y es la Consejería de Medio Ambiente la que pone en la actualidad más "pegas", sin embargo la reforma efectuada sobre el suelo no urbanizable (Modificación Ley del Suelo 4/2000) está pensada para poder urbanizar más territorio, con menos trabas.

La salud de la Tierra es una tarea común y compartida. Este planeta es nuestro único hogar y si queremos detener su deterioro ambiental, hay que reconsiderar seriamente nuestros modos de vida urbana, desde el centro histórico a la periferia integrada en su territorio.

Son muy pocos los ejemplos que se han llevado a cabo con criterios de sostenibilidad en España y Andalucía. Casi todo se queda en buenas intenciones. Quizá, y eso es alentador, hay más preocupación entre las nuevas generaciones. Ya no hay vuelta atrás. 